\title{
Engineering, differentiation and harvesting of human adipose-derived stem cell multilayer cell sheets
}

\author{
Joan Oliva*,1,2, Arjie Florentino1, Fawzia Bardag-Gorce ${ }^{1}$ \& Yutaka Niihara ${ }^{1,2}$ \\ ${ }^{1}$ Department of Medicine, LA BioMed at Harbor UCLA Medical Center, Torrance, CA 90502, USA \\ ${ }^{2}$ Emmaus Life Sciences, Inc., 21250 Hawthorne Blvd., Suite 800, Torrance, CA 90503, USA \\ *Author for correspondence: Tel.: +424 571 7639; joliva@emmauslifesciences.com
}

\begin{abstract}
Aim: The study goals were to engineer and harvest scaffold-free undifferentiated/differentiated multilayer human adipose-derived stem cell (hADSC) cell sheets, in absence of treatment. Materials \& methods: The hADSC are seeded in $35 \mathrm{~mm}$ culture dishes. At confluence or when multilayer cell sheets are formed, hADSC are treated with predefined differentiation culture media (adipocyte, chondrocyte and osteoblast). Results: Undifferentiated hADSC and differentiated adipocyte, osteoblast and chondrocyte hADSC multilayer cell sheets (hADSCmCS) have been harvested. Hematoxylin \& eosin showed the formation of multilayer cell sheets. Undifferentiated hADSC multilayer cell sheets preserve their stem cell markers. Differentiated adipocyte, osteoblast and chondrocyte hADSCmCS expressed specific markers. Conclusion: This simple protocol opens possibilities to engineer scaffold-free hADSCm cell sheet to transplant them on damaged organs.
\end{abstract}

Graphical abstract: Graphic showing the different possibilities to use adipose-derived stem cells multilayer cell sheets.

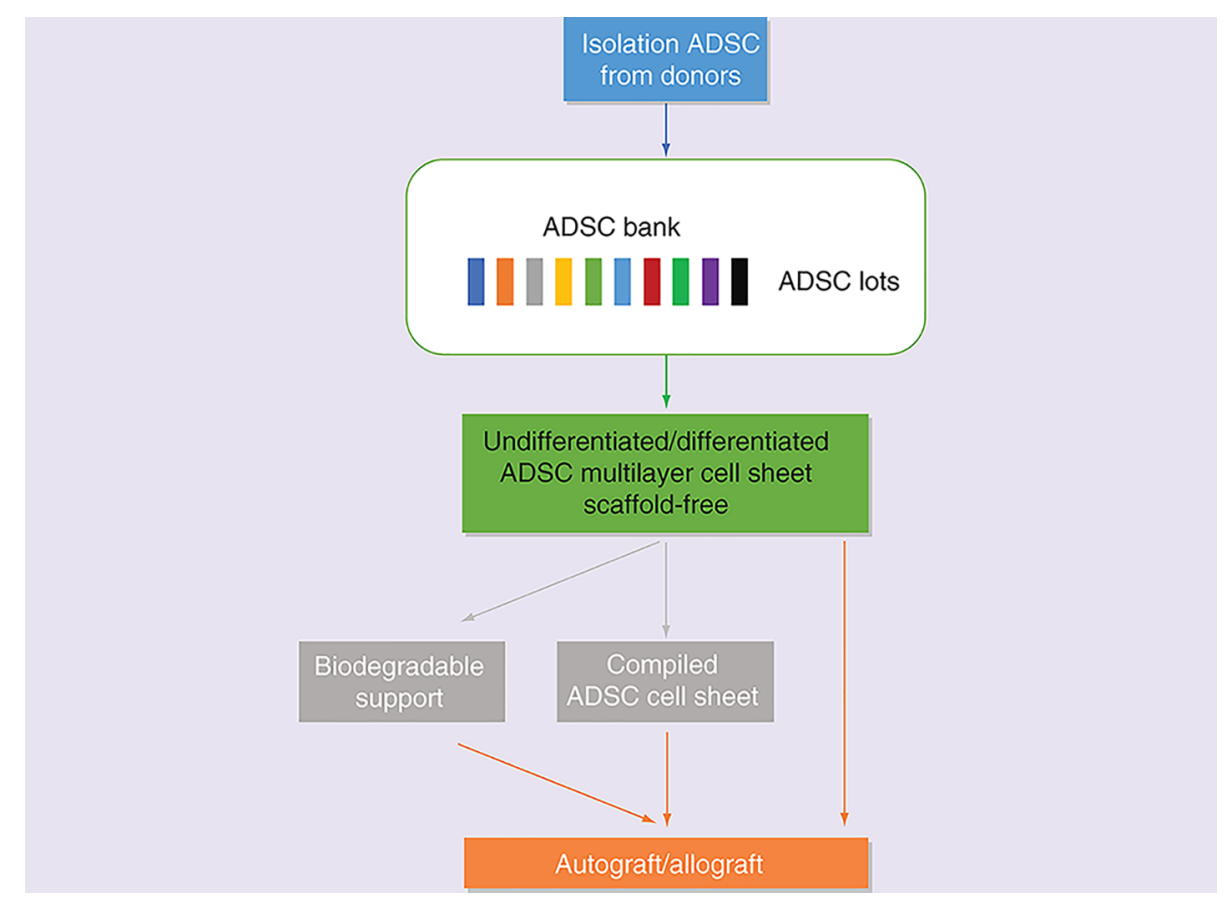

First draft submitted: 2 May 2018; Accepted for publication: 5 February 2019; Published online: 4 March 2019

Keywords: adipose-derived stem cells $\bullet$ cell therapy $\bullet$ differentiation $\bullet$ gene therapy $\bullet$ harvesting $\bullet$ multilayer cell sheet 
Stem cells can be classified in different groups, based on their origin, engineering and potential to differentiate: embryonic, totipotent, multipotent and induced pluripotent stem cells [1]. Adipose-derived stem cells (ADSC) are pluripotent mesenchymal stem cells (MSC) with the potential of self-renewal and the potential to differentiate in any type of cells such as adipocyte, myocytes and neurons [1]. In 2006, Dominici et al. defined three minimal criteria for adult MSC [2]: adherence to a plastic surface, specific surface antigen expression and multipotent differentiation potential. In addition to these criteria, other factors must be considered in the stem cells selection, such as the use of a gentle, surgically invasive procedure to isolate stem cells and the isolation of a high number of MSC, which is the case for ADSC isolation. In addition, there are no ethical issues in using ADSC, contrary to the case for embryonic stem cells.

Injection of stem cells can be enough to reverse a disease such as it was proven with the bone marrow transplantation. Based on the same approach, before or after differentiation, human adipose-derived stem cell (hADSC) are usually directly injected in organs, making it very difficult to control their migration; therefore, the cell therapy efficacy [3]. Depending on the choice of vein for intravenous injection, stem cells can migrate into different organs in different numbers, but they mainly target the lungs [4]. For the liver, MSC were injected in the hepatic artery to decrease cell diffusion and ectopic migration [5]. Additionally, their survival rate is very low (from 0.01 to 6\%), which thus decreases the efficacy of the stem cell therapy, on the targeted organ [6]. In addition, injected MSC disappeared at a very high rate after injection [7]. The injection of isolated MSC is a random treatment to target damaged tissue; however, it is also the easiest methodology to inject stem cells into the body. It was proven that the injection of stem cells in the heart improved its function. But a recent publication showed that the grafting of a stem cells cell sheet directly on the infarcted area resulted in a better improvement of the heart functionality, compared with the injection of the same cells into the heart [8]. The authors used a cell-seeded Gelfoam scaffold, made of collagen (Pharmacia \& Upjohn Kalamazoo, MI, USA), to graft the MSC cell sheet. Similar observation was reported on the meniscus regeneration. Katagiri et al. reported that the transplantation of MSC on the meniscus were more efficient than MSC injection [9]. Grafting layer(s) of cells can overcome the random migration of cells and increase their treatment efficacy by targeting and maintaining a higher number of cells in the desired area. Different scaffolds were developed to graft directly the cells on the tissue [10].

The type of stem cells and the compounds used to differentiate the stem cells are very important factors, but in addition tissue engineering requires the development of a carrier or scaffold for the transplantation. Different scaffolds were developed using natural and synthetic biomaterial such as polyethylene glycol, ceramic, collagen and hyaluronan [11]. Scaffold chemical and physical properties must be considered to treat each disease or organs, to maintain or to support the stemness and differentiation properties of stem cell. Others parameters such as rate of scaffold degradation, the type of connection (polymer chain connected linearly, branched or just crosslinked), flexibility are important [10]. For example, because of the cartilage location, semisolid scaffolds were used to graft the stem cells in the joint such as hydrogel, poly(lactic-co-glycolic) acid (PLGA). Franklin et al. injected MSC in the articulation of osteoarthritis and cartilage Beagles model and observed an improvement in their lameness [12,13]. The scaffold can have a dual function: as a support to graft the cells on the damage tissue but it can also support stem cell differentiation. The culture of bone marrow stem cells in a fribin-poly (ester-urethane) increased the differentiation of the bone marrow stem cells into chondrogenic cells because the mechanical movement of the scaffold mimics the natural joints movement [14].

It was demonstrated that stem cells can grow in different types of scaffolds and could differentiate into different type of cells. To confirm the differentiation properties of ADSC (based on Dominici criteria [2]), differentiated ADSC is usually confirmed with dye staining such as Red oil for adipocyte, Alizarin red for osteoblast and Alcian blue for chondrocyte. In addition, their differentiation must be confirmed by the detection of cell type protein expression. Choi et al. differentiated human ADSC on a silk scaffold into the adipocyte and confirmed the expression of LEP (leptin) by the adipocyte [15]. The differentiated ADSC into bone expressed specific bone proteins such as SPP1 (osteopontin), COL1, ALPL and RUNX2 [16,17]. The chemical composition of the scaffold plays a critical role in the ADSC differentiation. Adipose derived stem cells differentiated with higher rate in a fibrin glue scaffold than in poly D, L-lactic glycolic acid (PLGA) or alginate scaffold [18]. These results were confirmed with expression of chondrocyte specific markers such as COL1, COL2, SOX9 and ACAN.

It was reported that scaffolds used to repair the tissues can be the trigger of an immunoreaction [19,20], which can be avoided when using a scaffold-free protocol. Scaffold-free is a great approach to graft the cells directly on 
the tissue. The scaffold-free methodology can be performed if cells naturally form tight inter-cellular connection, strong enough to allow the cell sheet to be lifted. In 2004, Nakamura et al. engineered a multilayer epithelial cell sheets and grafted it directly on the damaged corneas of rabbit [21]. Using a defined cell culture media and in presence of feeder cells, progenitor oral mucosa epithelial cells differentiated and formed a strong cell sheet. However, they had to use a thermoresponsive surface to harvest the multilayer cell sheet, which can be used also for monolayer cell sheets. Other methodologies were used to harvest cell sheet such as Ethylenediaminetetraacetic acid treatment [22] or using saline solution [23], only for cell sheets with a strong cell-cell connection. We propose a simple methodology to engineer and harvest cell sheet, using ADSC, with a scaffold-free methodology. Besides the simplification of the protocol for cell sheet harvesting, the use of scaffold-free is an important parameter regarding the immune system activation.

\section{Material \& methods}

\section{ADSC cell culture}

Human ADSC cells were purchased from Life Technology (Life Technology, MA, USA). The cells were cultured and passaged following the manufacturer's instructions (Life Technology) using the culture media MesenPRO RS ${ }^{\text {TM }}$ Medium (Life Technology). The hADSC were seeded at $2.2 \times 10^{4}$ cells $/ \mathrm{cm}^{2}$ for amplification. The hADSC at passage 3 were used for the differentiation and harvesting protocol (Figure 1).

\section{Culture, differentiation of hADSC}

The hADSC cells were seeded in $35 \mathrm{~mm}$ cell culture dishes (Corning, Inc., NY, USA) at $2.2 \times 10^{4} \mathrm{cells} / \mathrm{cm}^{2}$. The hADSC differentiation was performed following the manufacturer's instructions (Life Technology). At 1011 days, the hADSC had formed multilayer cell sheet, which was harvested from the $35 \mathrm{~mm}$ dish (Figure 1). We used the StemPro ${ }^{\circledR}$ Adipogenesis Differentiation Kit, StemPro Osteogenesis Differentiation Kit and StemPro ${ }^{T M}$ Chondrogenesis Differentiation Kit (Life Technology). Adipogenesis, osteogenic and chondrogenic treatment on hADSC started when the hADSC reached confluence and when the hADSC formed a multilayer cell sheet (Figure 1). Details of the multilayer cell sheet cell culture are explained in the Figure 1. Adipocyte hADSC multilayer cell sheets were harvested 19 days after initial seeding, osteogenic hADSC multilayer cell sheets were harvested 30 days after initial seeding, and chondrocyte hADSC multilayer cell sheets were harvested 15 and 23 days after initial seeding.

\section{Harvesting of hADSC}

To harvest the multilayer cell sheets, we used a polyvinylidene difluoride (PVDF) membrane and forceps. The PVDF membranes were cut in a donut shape (PVDF, hydrophilic, $5.0 \mu \mathrm{m}, 47 \mathrm{~mm}$, white, plain, 100; Burlington, MA, USA) with the following size: $16 \mathrm{~mm}$ outer diameter and $9 \mathrm{~mm}$ inner diameter. The culture media was removed, and the cell sheets were washed with phosphate-buffered saline (PBS). The PBS was removed and replaced by fresh PBS. Forceps were used to detach the edges of the cells sheets and wrapped on the PVDF membrane placed in the center of the cell sheet. Once the edges were wrapped over the PVDF membrane, the whole PVDF/cell sheet was lifted with the forceps and placed in another $60 \mathrm{~mm}$ cell culture dish (Corning, Inc.), containing PBS. The cell sheets were unwrapped, and they were fixed in $10 \%$ Neutral buffered formalin for immunohistochemistry staining.

\section{Hematoxylin \& eosin \& immunocytochemistry staining}

Engineered cell sheets were fixed in $10 \%$ neutral buffered formalin and embedded in paraffin. Tissue sections were then stained with hematoxylin \& eosin (H\&E) or used for immunofluorescent staining with CD19, CD73, AcetylCoA1 (NovusBIo, CO, USA), CD29, COLII (Collagen II), HLA-A, HLA-DR (Abcam, MA, USA), CD45R, CD105, BGLAP (Osteocalcin), SREBP1 (ThermoFisher Scientific, CA, USA), PPARg (Cell Signaling, MA, USA), ACAN (Aggrecan) and SPARC (ThermoFisher Scientific). Alexa Fluor ${ }^{\circledR} 488$ donkey antimouse conjugated second antibodies, Alexa Fluor 488 donkey antirabbit conjugated second antibodies and Alexa Fluor 488 donkey antirat conjugated second antibodies (Invitrogen, OR, USA) were used. Propidium iodine (Invitrogen) was used to stain nuclear DNA. A Nikon 400 fluorescence microscope was used to analyze the slides (Nikon Inc., NY, USA).

\section{Results}

By letting the hADSC grow for an extra period of time after reaching confluence, the density of hADSC increased rapidly, resulting in the formation of a multilayer cell sheet (Figure $2 \mathrm{~A} \& \mathrm{~B}$ ) and confirmed by a H\&E staining 
(A)

\section{ADIPOCYTE DIFFERENTIATION}

ADSC $2 \times 10^{5}$ cells seeded

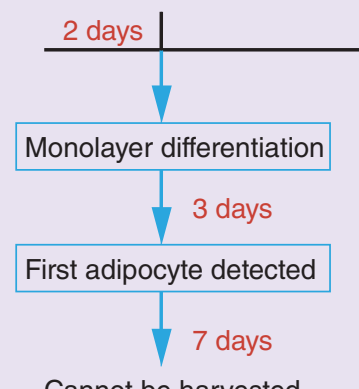

Cannot be harvested

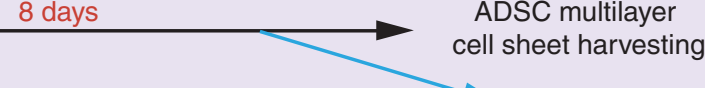

Multilayer differentiation

1 days

First adipocyte detected

9 days

Harvested

(B)

OSTEOGENIC DIFFERENTIATION

ADSC $2 \times 10^{5}$ cells
seeded

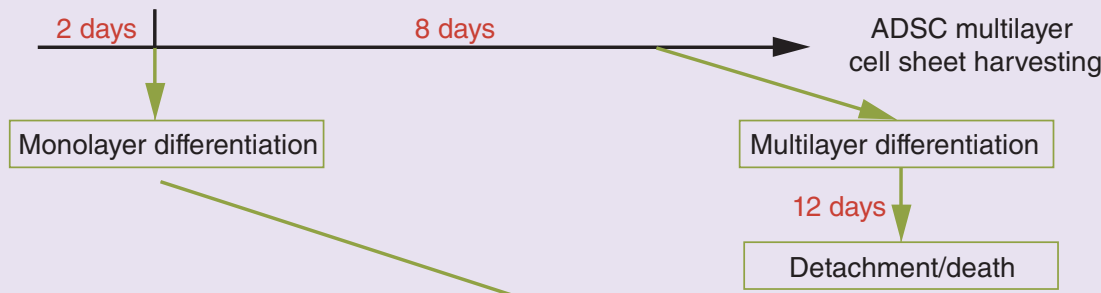

ADSC grow and differentiate at the same time

28 days

Harvested

(C)

\section{CHONDROGENIC DIFFERENTIATION}

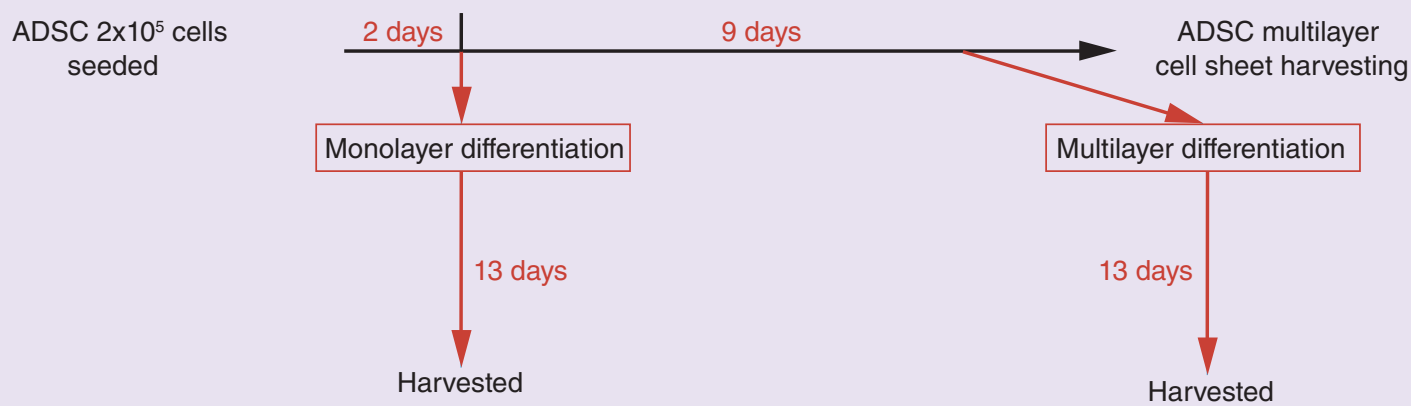

Figure 1. Summary of the human adipose-derived stem cell growth and treatment. (A-C) The timeline of undifferentiated hADSC cell sheet culture. (A) The timeline to engineer an adipocyte multilayer cell sheet. (B) The timeline to engineer an osteoblast multilayer cell sheet. (C) The timeline to engineer a chondrocyte multilayer cell sheet.

ADSC: Adipose-derived stem cell; hADSC: Human adipose-derived stem cell.

(Figure 2D). Only 10 days in culture was necessary to engineer undifferentiated hADSC multilayer cell sheets for harvest (Figure 2C). The formation of the multilayer cell sheet did not affect the expression of hADSC markers CD19-, CD45R ${ }^{-}, \mathrm{CD} 29^{+}, \mathrm{CD}^{-} 3^{+}$and $\mathrm{CD} 105^{+}$(Figure 2E). The ability to engineer undifferentiated multilayer hADSC cell sheets created the opportunity to engineer and harvest differentiated multilayer hADSC cell sheets. Undifferentiated monolayer and multilayer hADSC cell sheets were treated with adipocyte, osteogenic 


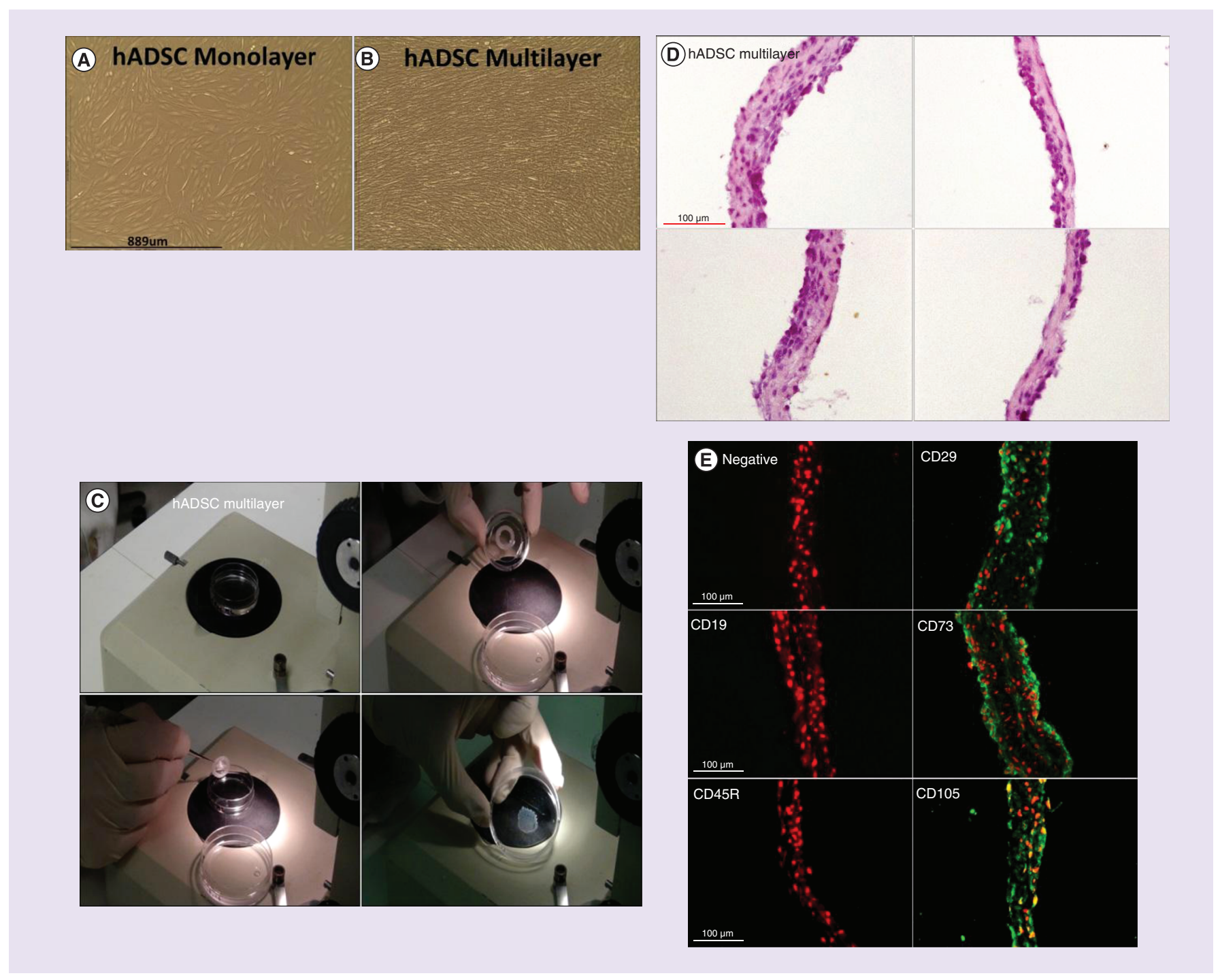

Figure 2. Summary of the undifferentiated human adipose-derived stem cell cell sheets. (A) hADSC monolayer, when the adipocyte, osteoblast and chondrocyte differentiation started (scale bar: $889 \mu \mathrm{m})$. (B) hADSC multilayer, the day of the harvesting. Density of cells is much higher than monolayer. (C) Harvesting steps of the undifferentiated multilayer hADSC cell sheet. (D) Hematoxylin \& eosin staining shows the morphology of the undifferentiated hADSC cell sheet. (E) Immunostaining of the cell sheet shows hADSC preserving the expression of positive markers of hADSC cells (CD29, CD73 and CD105), and negative for CD19 and CD45R.

hADSC: Human adipose-derived stem cell.

and chondrocyte culture media, which were purchased from the manufacturer, following the timing described in Figure 1.

Total 2 days after reaching confluence, undifferentiated hADSC monolayers were treated with adipocyte culture media, and 8 days later, undifferentiated hADSC multilayer cell sheets were treated with adipocyte culture media. The hADSC monolayer cell sheet differentiated into adipocyte cells, but their harvesting was not possible because the cell sheet was weak. The hADSC multilayer cell sheet treated 10 days with adipocyte culture media were harvested (Figure 3B \& C). The H\&E of the adipocyte multilayer sheets showed the morphology of the adipocyte cell sheet (Figure 3D). Staining showed that specific markers of adipocytes, such as Acetyl-CoA1, SREBP1 and PPARg, were expressed in the adipocyte hADSC cell sheet multilayers (Figure 3E), confirming the differentiation of hADSC into adipocytes [24].

Undifferentiated multilayer hADSC could not have differentiated into osteogenic multilayer hADSC cell sheets because hADSC detached spontaneously and died during osteogenic treatment (data not shown). However, os- 

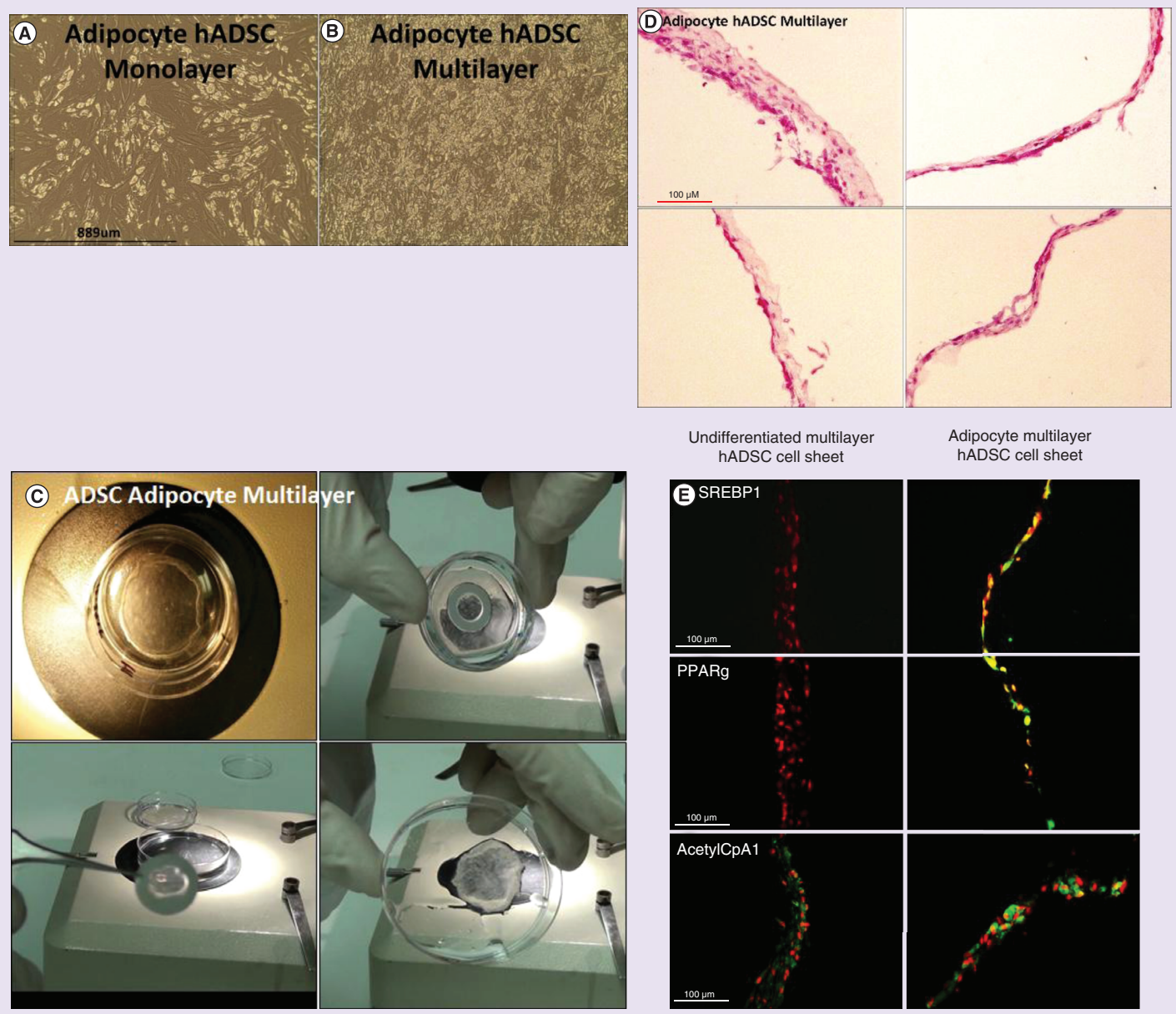

Figure 3. Summary of the adipocyte multilayer human adipose-derived stem cell cell sheets. (A) Monolayer of adipocyte hADSC cell sheet, the last day of treatment (scale bar: $889 \mu \mathrm{m}$ ). (B) Multilayer of adipocyte hADSC, the last day of treatment. Density of adipocyte in the multilayer cell sheet is much higher than monolayer. (C) Steps for harvesting the adipocyte multilayer hADSC cell sheet. (D) Hematoxylin \& eosin staining shows the morphology of the adipocyte hADSC cell sheet. (E) Immunostaining of the adipocyte cell sheet shows that the cell sheet increases the expression of adipocyte markers such as SREBP1, PPARg and AcetylCoA1. ADSC: Adipose-derived stem cells; hADSC: Human adipose-derived stem cell.

teogenic multilayers of hADSC cell sheets were engineered by treating hADSC monolayer because hADSC were still growing, increasing the cell density and the cell-cell contact, during osteogenic differentiation. After a total of 28 days after osteogenic differentiation treatment started, osteogenic multilayer hADSC cell sheets were harvested (Figure 4C). The H\&E staining showed the morphology of a multilayer cell sheet (Figure 4D). Cell identities were confirmed by immunohistostaining with osteocalcin, a specific marker of osteoblasts (Figure 4E).

Multilayer chondrocyte cell sheets were engineered when the hADSC monolayer cell sheets were treated with chondrocyte differentiation culture media provided by the manufacturer. In Figure 5B, a high density of chondrocytes was observed 9 days after the initiation of differentiation treatment. The cells formed a strong cell-cell connection, making harvesting possible (Figure 5C). In Figure 5D, H\&E staining shows the morphology of the multilayer chondrocyte cell sheet. In Figure 5E, the expression of specific markers of chondrocytes was confirmed; the chondrocyte cell sheet stained positively for ACAN, SPARC and COLII when the undifferentiated multilayer cell sheets were negative.

Also, the expression of histocompatibility antigens HLA-A and HLA-DR was analyzed on the hADSC multilayer cell sheets. The formation of multilayer hADSC and culture conditions did not affect the expression of HLA-A and HLA-DR. We confirmed that HLA-A is still expressed in undifferentiated hADSC and adipocyte, osteogenic and chondrocyte hADSC multilayers (Figure 6A-D) and that HLA-DR is not (Figure 6E-H). These results indicate 

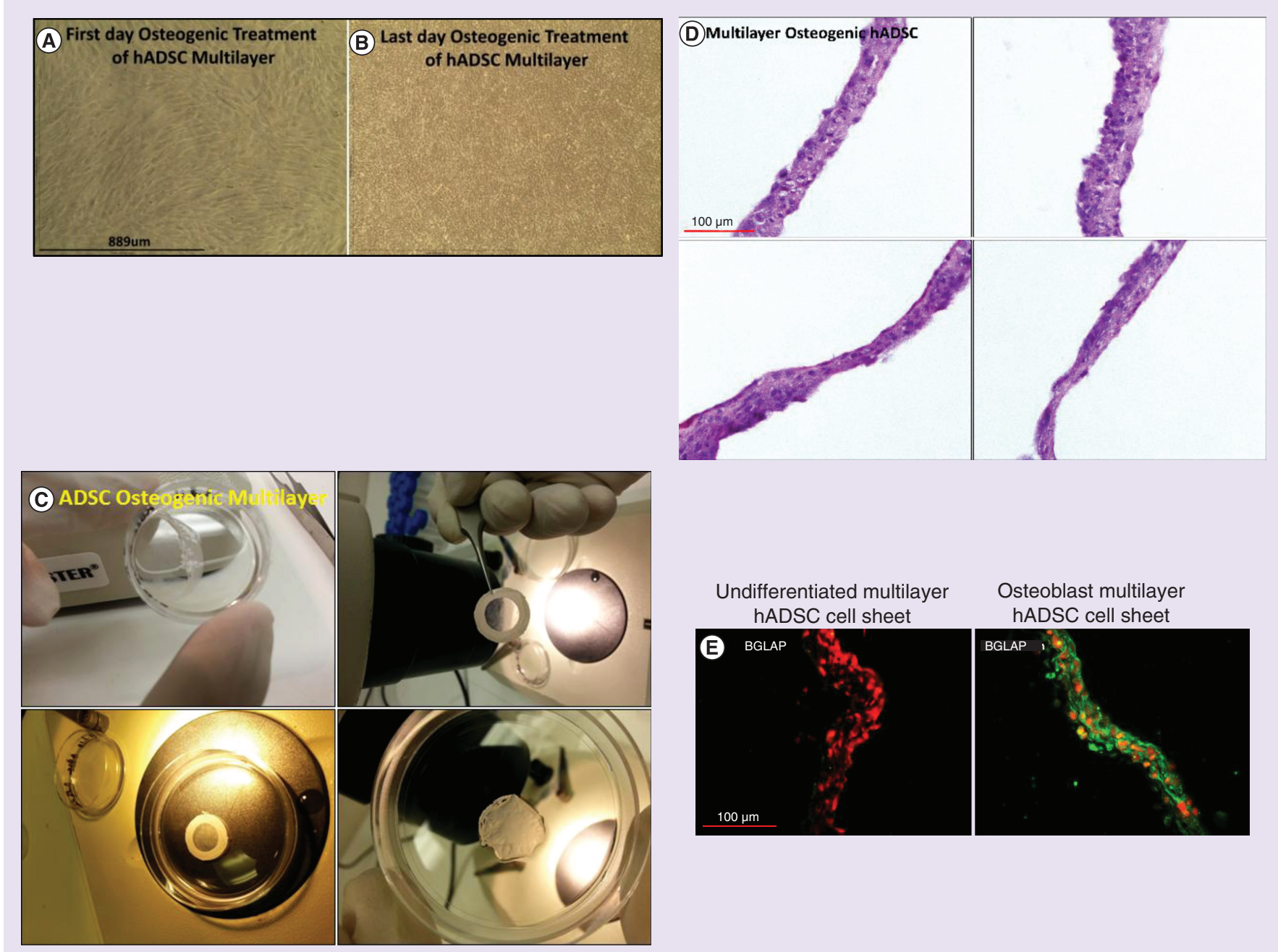

Osteoblast multilayer
hADSC cell sheet

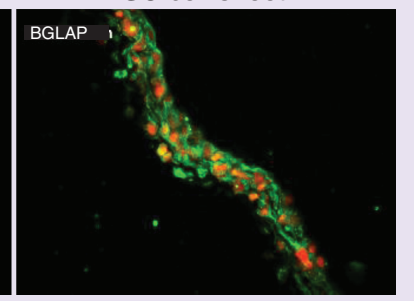

Figure 4. Summary of the osteogenic human adipose-derived stem cell multilayer cell sheet. The first (A) and last day (B) of osteogenic treatment (scale bar: $889 \mu \mathrm{m}$ ). Density of osteogenic hADSC cells is high. (C) Step for harvesting the osteogenic multilayer hADSC cell sheet. In (D), Hematoxylin \& eosin staining shows the morphology of the osteogenic multilayer hADSC cell sheet. (E) Immunostaining of the osteogenic cell sheet shows that the cell sheet increases the expression of osteogenic markers such as BGLAP.

ADSC: Adipose-derived stem cells; hADSC: Human adipose-derived stem cell.

that undifferentiated or differentiated hADSC multilayer cell sheets can be used on patients as autologous grafts or allografts.

\section{Discussion}

The production of organs based on stem cells is a very difficult challenge. Initial research was based on the differentiation of stem cells in culture into a specific cell type. The ADSC are typically used in a single cell solution that is injected into targeted organs or in the bloodstream upstream of the targeted organ [3]. The injection of these cells was supposed to reverse the disease phenotype, but its efficacy was low or was only effective in the short term [25], even though the injection of ADSC seemed to be safe with no life threatening effects or tumor formation [26]. Collagen was one of the first biological compounds used to harvest a monolayer of keratinocytes that were transplanted into the oral mucosal cavity [27]. Human corneal endothelial cell sheets were also engineered by using collagen sheets as a support for harvesting in a rabbit model [28]. Menasche's group showed in 2011 that grafting a monolayer ADSC cell sheet on the heart improves the survival of postinfarction rats more than the injection of single ADSC cells [8]. A similar conclusion was reported by Dekaris who grafted a multilayer amniotic membrane onto corneal ulcers [29]. These results confirmed the advantage of cell sheets over the injection of isolated 

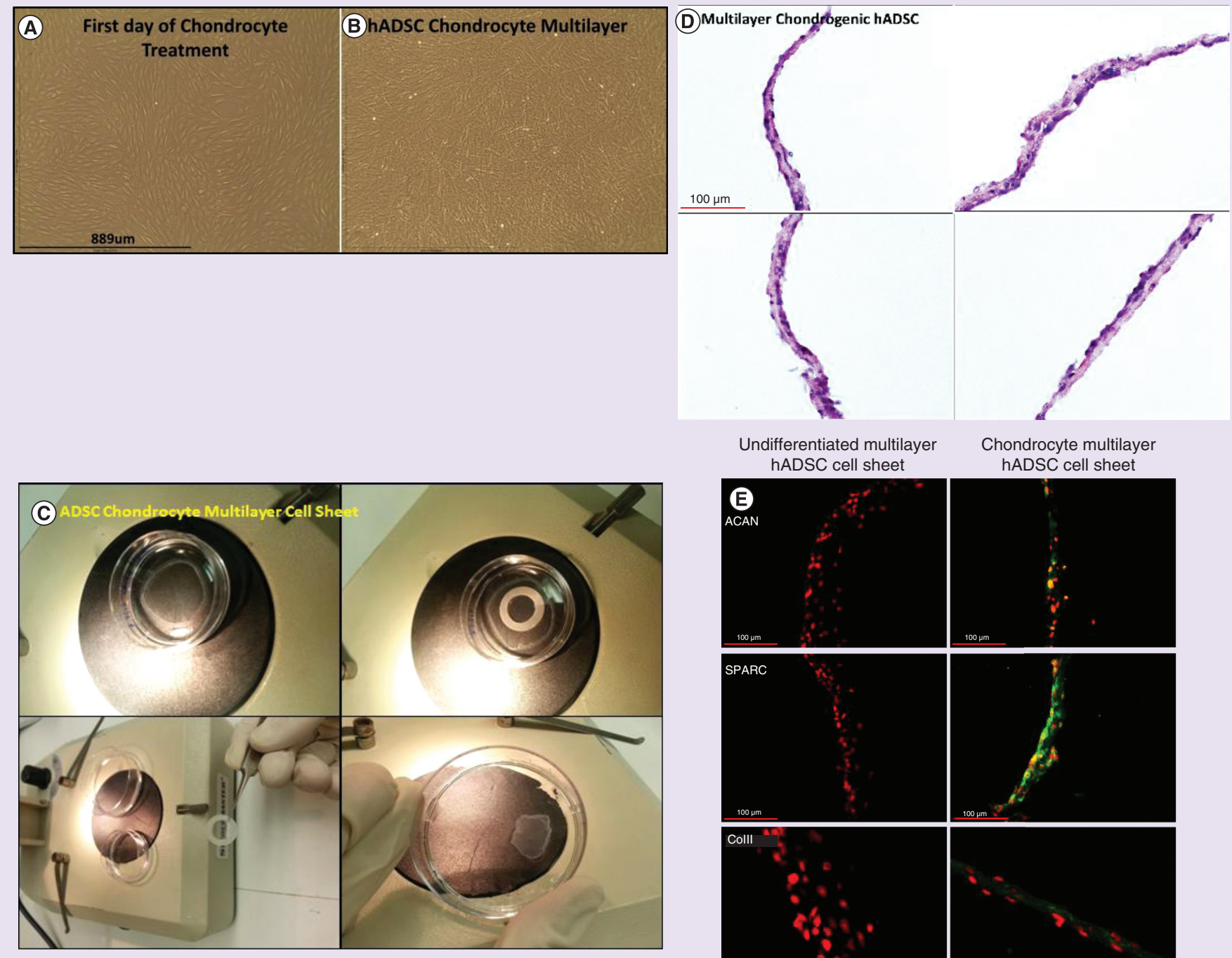

Chondrocyte multilayer
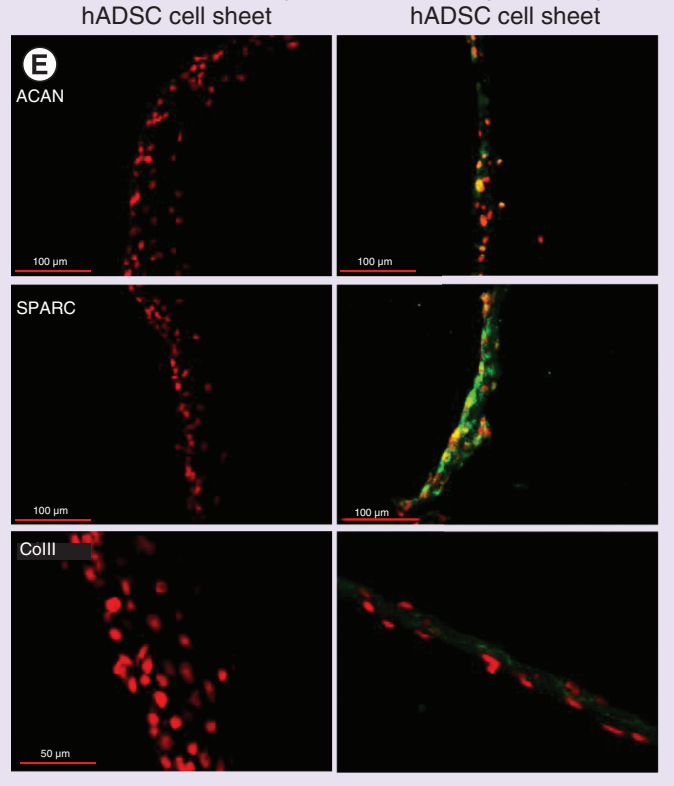

Figure 5. Summary of the chondrocyte human adipose-derived stem cell multilayer cell sheet. The first (A) and last day (B) of chondrocyte treatment (scale bar: $889 \mu \mathrm{m}$ ). Density of chondrocyte hADSC cells is high. (C) Steps for harvesting the chondrocyte multilayer hADSC cell sheet. (D) Hematoxylin \& eosin staining shows the morphology of the chondrogenic multilayer hADSC cell sheet. (E) Immunostaining of the chondrogenic cell sheet shows that the cell sheet increases the expression of chondrocyte markers such as ACAN, SPARC and COLII.

hADSC: Human adipose-derived stem cel.

cells for curing damaged organs. For both studies, the authors used a thermo-sensitive surface to harvest the cell sheet [8] or they used the amniotic membrane [8,29]. Other methodologies were used to harvest cells sheets such as EDTA, dispase, magnetic force, electron polarization, $\mathrm{pH}$ decrease, UV illumination or hexacyanoferta(II)-induced dissolution [22,30]. The use of chemical or specific surface to harvest the cells complicates translation application for human studies because of the regulations.

Our group has developed an easy and efficient method to engineer and harvest hADSC multilayer cell sheets (undifferentiated, adipocyte, osteogenic and chondrogenic), with the possibility of grafting an entire multilayer cell sheets on targeted organs or support (allograft or xenograft). However, we were not able to engineer a multilayer osteogenic hADSC cell sheet from undifferentiated multilayer hADSC, but it was engineered from monolayer hADSC (Figure 1). This result indicates that a specific protocol must be developed for a specific differentiation based on the confluence of the hADSC, initial cell density, the capacity of the cells to form a strong cell-cell contact 


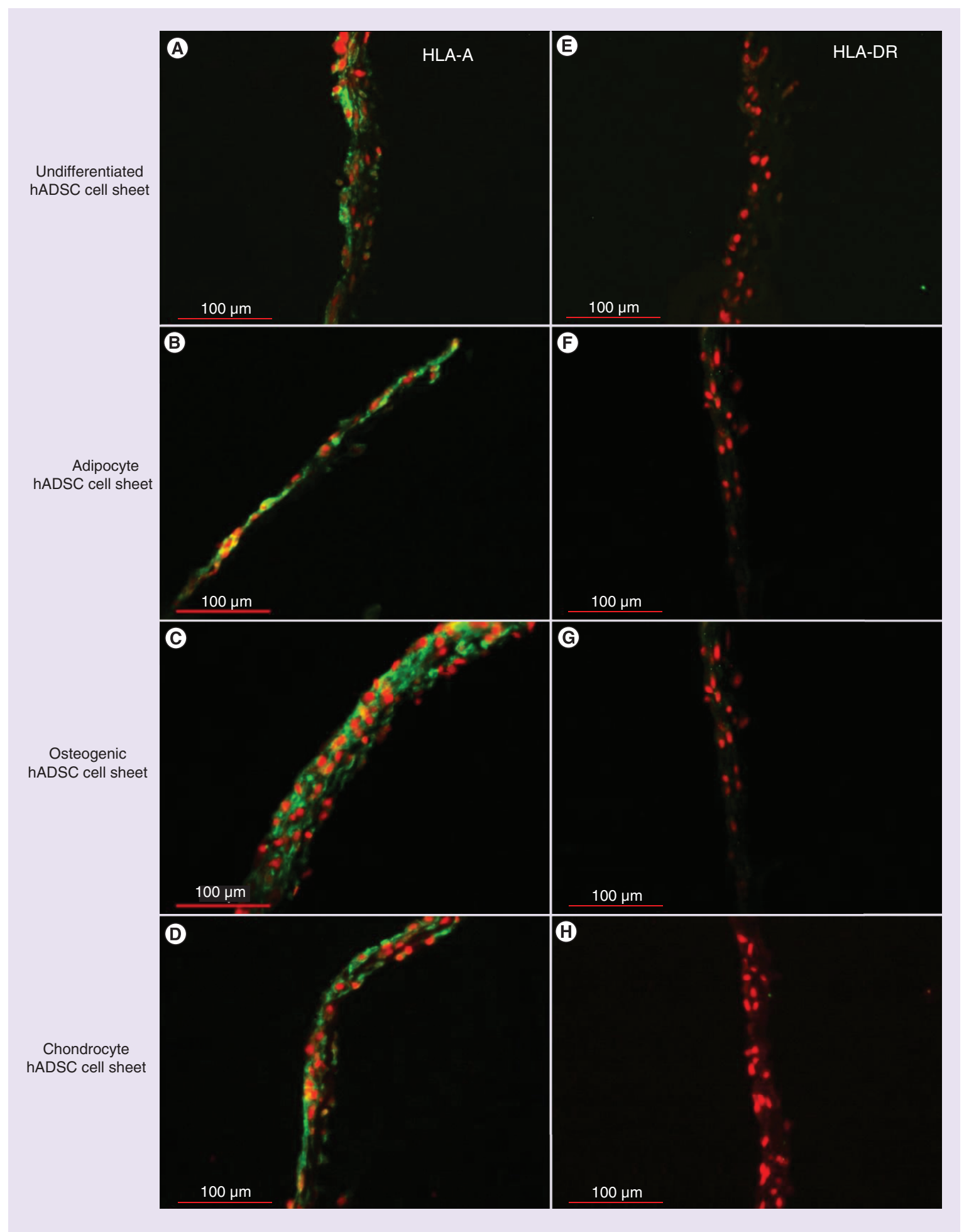

Figure 6. Expression of the Histocompatibility complex in the human adipose-derived stem cells multilayer cell sheet. (A-D) Histocompatibility antigen HLA-A was detected in undifferentiated, adipocyte, osteogenic and chondrocyte hADSC multilayer cell sheets. (E-H) Histocompatibility antigen HLA-DR was not detected in the undifferentiated, adipocyte, osteogenic and chondrocyte hADSC multilayer cell sheets.

hADSC: Human adipose-derived stem cell. 
and the duration of the differentiation treatment [31]. Cell-cell contact is an important parameter to engineer and harvest multilayer cell sheets. This parameter can be a limiting factor for the development of multilayer cell sheets because not all type of cells can form a strong cell-cell connection. Also, cell-cell contact or loss of contact can modify the cells phenotype and their proliferation/differentiation characteristics [32-34]. We confirmed that undifferentiated multilayer cell sheet preserved their stemness, especially the absence of HLA-DR, a key factor for allograft or xenograft. Because of the absence of HLA-DR, human ADSC have been tested in different animals in absence of adverse events reported: mouse, rat, rabbit, zebrafish, swine and dog [35]. The ADSC are a major and abundant source of stem cells, easy to isolate with a noninvasive method. A small ADSC biopsy can provide billions of cells after mass production [36]. Another limiting factor for the use of human ADSC is that their differentiation potential decreases with the number of passages [37]. Banks of hADSC can be built to store different human ADSC (low passage) based on their genotype, differentiation properties and their capacity to reverse diseases [38]. These hADSC banks will provide enough cells for clinical applications all over the world in the field of cell and gene therapy because the absence of histocompatibility complex will allow allografting. Furthermore, large hADSC banks will be composed of hADSC with different genotype. It is important to remember that, before stem cells are used for patients treatment, their genome must be sequenced to ensure the regenerative capacity and safety of stem cells [39].

Our study does however have a number of limitations. First, only four different types of ADSC cell sheet were developed, and specific differentiation protocol must be defined for other types of cells (myocytes, hepatocytes, epithelial cells, etc). We were also unable to control the number of layers forming the cell sheet, and the influence of the number of layers on the cell sheet therapeutic properties is unknown. In addition, our study was an in vitro study, and the regenerative function on an in vivo study was not performed. Despite these limitations, the present study demonstrated an easy method to engineer graftable, scaffold-free cell sheets.

\section{Translational perspective}

The hADSC cell sheet products have been shown to have better effects than the injection of single stem cells, as previously mentioned. The hADSC have an advantage over bone marrow stem cells in that they can be obtained very easily from patients (from donors or from liposuction which are usually discarded), making them an almost unlimited source of adult stem cells. We hope that the simplicity of the protocol presented might benefit laboratories and companies involved in the development of multilayer hADSC cell sheet products and be beneficial to the mass production of cell sheets. In the future, this could lead to the ability of two or more different hADSC cell sheets containing different differentiated (or not) cell types to be produced together and for one sheet to be compiled on top of the other one.

We demonstrated here that multilayer hADSC cell sheets maintain their differentiation capacity, indicating their ability to differentiate into desired cell types. This could potentially be utilized in the future, such as in the repair of articulated limbs, which is a highly complex process due to the involvement of the bone and the cartilage. In theory, hADSC multilayer cell sheets differentiated into chondrocytes could be placed on the top of an osteoblast cell sheet before being grafted on to the damaged articulation areas. The healing of skin wounds, such as those seen in diabetic patients, could also potentially be accelerated through the grafting of a whole multilayer cell sheet directly on to the wound. The use of human ADSCs should also decrease the local inflammatory response, and the hADSCs could differentiate into the cells needed to heal the wound, such as keratinocytes, but also release factors involved in tissue repair.

This simple approach could potentially also be utilized alongside 3D printing technology for regenerative medicine applications. Our approach was performed on a circular flat surface, but it is possible to conduct similar tests by seeding hADSC cells directly onto a support of any shape or dimension. We therefore propose that a support printed in the shape of a particular organ or tissue could be wrapped with specific types of cell sheet. For example, human ADSC could be differentiated into endothelial cells sheets and smooth muscle cells and wrapped on a blood vessel support shape. In theory, a 3D-printed support in the shape of a patient's bone could also be produced, perhaps through the use of bone imaging technology to ensure it is created with the correct shape and size in order to fit a specific patient. The hADSCs could then be seeded onto the support and using a specific culture media, the hADSCs could be differentiated into an osteoblast multilayer cell sheet and the scaffold could then be grafted into the patient. In addition to the applications already mentioned, there is potential application in the field of plastic surgery using the the scientific methodologies for bone reconstruction, burn care by combining the stem cells, the scaffolds and the differentiation protocols. 
Before projects utilizing hADSC, multilayer cell sheets can be translated to patient applications; however, it will be important to confirm the functionality of differentiated cell sheets in vivo, by testing them on animal disease models.

\section{Summary points}

Injection of isolated stem cells is a concern for ectopic migration \& efficacy

- Injected isolated stem cells in a body can migrate anywhere over the body, settle and impair the organ/tissue function by growing, for example as a tumor.

- Injected isolated stem cells are less efficient to reverse the disease phenotype than a grafted cell sheet.

Engineering of multilayer cell sheet with adult human adipose-derived stem cells

- Overextended cell culture time of human adipose-derived stem cell (hADSC) showed the hyperconfluence of the hADSC over the cell culture dishes, in absence of treatment, and the formation of a multilayer hADSC cell sheet.

- Treatment of hADSC with differentiation culture media differentiated into adipocyte, chondrocyte or osteoblast multilayer hADSC.

Harvesting of undifferentiated or differentiated into adipocyte, chondrocyte or osteoblast hADSC multilayer

- The hADSC built a strong cell-cell connection, allowing the harvesting of the multilayer cell sheets.

- Undifferentiated and differentiate hADSC multilayer cell sheets were harvested, in absence of chemical pretreatment or the use of pretreated cell culture dish.

- A scaffold-free multilayer cell sheet was harvested.

Financial \& competing interests disclosure

This work was supported by Emmaus Medical, Inc. (Torrance, CA, USA). Y Niihara is the Chairman of the Board of Directors and CEO of Emmaus Medical, Inc, and J Oliva is employee of Emmaus Medical, Inc. Medical writing support was provided by American Journal Experts and was funded by Emmaus Medical, Inc. The authors have no other relevant affiliations or financial involvement with any organization or entity with a financial interest in or financial conflict with the subject matter or materials discussed in the manuscript apart from those disclosed.

No writing assistance was utilized in the production of this manuscript.

\section{Open access}

This article is distributed under the terms of the Creative Commons Attribution License 4.0 which permits any use, distribution, and reproduction in any medium, provided the original author(s) and the source are credited. To view a copy of the license, visit http://creativecommons.org/licenses/by/4.0/

\section{References}

1. Miana VV, Gonzalez EAP. Adipose tissue stem cells in regenerative medicine. Ecancermedicalscience 12, 822 (2018).

2. Dominici M, Le Blanc K, Mueller I et al. Minimal criteria for defining multipotent mesenchymal stromal cells. The International Society for Cellular Therapy position statement. Cytotherapy 8(4), 315-317 (2006).

3. Fodor PB, Paulseth SG. Adipose derived stromal cell (ADSC) injections for pain management of osteoarthritis in the human knee joint. Aesthet. Surg. J. 36(2), 229-236 (2016).

4. Kurtz A. Mesenchymal stem cell delivery routes and fate. Int. J. Stem Cells 1(1), 1-7 (2008).

5. Sun L, Fan X, Zhang L et al. Bone mesenchymal stem cell transplantation via four routes for the treatment of acute liver failure in rats. Int. J. Mol. Med. 34(4), 987-996 (2014).

6. Gyongyosi M, Blanco J, Marian T et al. Serial noninvasive in vivo positron emission tomographic tracking of percutaneously intramyocardially injected autologous porcine mesenchymal stem cells modified for transgene reporter gene expression. Circ. Cardiovasc. Imaging 1(2), 94-103 (2008).

7. Urban VS, Kiss J, Kovacs J et al. Mesenchymal stem cells cooperate with bone marrow cells in therapy of diabetes. Stem Cells 26(1), 244-253 (2008).

8. Hamdi H, Planat-Benard V, Bel A et al. Epicardial adipose stem cell sheets results in greater post-infarction survival than intramyocardial injections. Cardiovasc. Res. 91(3), 483-491 (2011).

9. Katagiri H, Muneta T, Tsuji K et al. Transplantation of aggregates of synovial mesenchymal stem cells regenerates meniscus more effectively in a rat massive meniscal defect. Biochem. Biophys. Res. Commun. 435(4), 603-609 (2013).

10. Prakasam M, Locs J, Salma-Ancane K, Loca D, Largeteau A, Berzina-Cimdina L. Biodegradable materials and metallic implants: a review. J. Funct. Biomater. 8(4), doi:10.3390/jfb8040044 (2017). 
11. Willerth SM, Sakiyama-Elbert SE. Combining stem cells and biomaterial scaffolds for constructing tissues and cell delivery. In: StemBook. Harvard Stem Cell Institute, MA, USA (2008).

12. Franklin SP, Stoker AM, Bozynski CC et al. Comparison of platelet-rich plasma, stromal vascular fraction (SVF), or SVF with an injectable PLGA nanofiber scaffold for the treatment of osteochondral injury in dogs. J. Knee Surg. 31(7), 686-697 (2018).

13. Lin H, Cheng AW, Alexander PG, Beck AM, Tuan RS. Cartilage tissue engineering application of injectable gelatin hydrogel with in situ visible-light-activated gelation capability in both air and aqueous solution. Tissue Eng. Part A 20(17-18), 2402-2411 (2014).

14. Gardner OFW, Musumeci G, Neumann AJ et al. Asymmetrical seeding of MSCs into fibrin-poly(ester-urethane) scaffolds and its effect on mechanically induced chondrogenesis. J. Tissue Eng. Regen. Med. 11(10), 2912-2921 (2017).

15. Choi JH, Bellas E, Vunjak-Novakovic G, Kaplan DL. Adipogenic differentiation of human adipose-derived stem cells on 3D silk scaffolds. Methods Mol. Biol. 702 319-330 (2011).

16. Correia C, Bhumiratana S, Yan LP et al. Development of silk-based scaffolds for tissue engineering of bone from human adipose-derived stem cells. Acta Biomater. 8(7), 2483-2492 (2012).

17. Ojansivu M, Johansson L, Vanhatupa $S$ et al. Knitted 3D scaffolds of polybutylene succinate support human mesenchymal stem cell growth and osteogenesis. Stem Cells Int. 2018, 5928935 (2018).

18. Sheykhhasan M, Qomi RT, Kalhor N, Mehdizadeh M, Ghiasi M. Evaluation of the ability of natural and synthetic scaffolds in providing an appropriate environment for growth and chondrogenic differentiation of adipose-derived mesenchymal stem cells. Indian J. Orthop. 49(5), 561-568 (2015).

19. Badylak SF, Gilbert TW. Immune response to biologic scaffold materials. Semin. Immunol. 20(2), 109-116 (2008).

20. Boehler RM, Graham JG, Shea LD. Tissue engineering tools for modulation of the immune response. Biotechniques 51(4), 239-240, (2011).

21. Nakamura T, Inatomi T, Sotozono C, Amemiya T, Kanamura N, Kinoshita S. Transplantation of cultivated autologous oral mucosal epithelial cells in patients with severe ocular surface disorders. Br. J. Ophthalmol. 88(10), 1280-1284 (2004).

22. Hashimoto H, Tamaki T, Hirata M, Uchiyama Y, Sato M, Mochida J. Reconstitution of the complete rupture in musculotendinous junction using skeletal muscle-derived multipotent stem cell sheet-pellets as a 'bio-bond'. PeerJ 4, doi: 10.7717/peerj.2231 (2016)

23. Lui PP, Wong OT, Lee YW. Application of tendon-derived stem cell sheet for the promotion of graft healing in anterior cruciate ligament reconstruction. Am. J. Sports Med. 42(3), 681-689 (2014).

24. Moseti D, Regassa A, Kim WK. Molecular regulation of adipogenesis and potential anti-adipogenic bioactive molecules. Int. J. Mol. Sci. 17(1), doi: 10.3390/ijms17010124 (2016).

25. Zhang L, Coulson-Thomas VJ, Ferreira TG, Kao WW. Mesenchymal stem cells for treating ocular surface diseases. $B M C$ Ophthalmol. 15(Suppl. 1), 155 (2015).

26. Ra JC, Shin IS, Kim SH et al. Safety of intravenous infusion of human adipose tissue-derived mesenchymal stem cells in animals and humans. Stem Cells Dev. 20(8), 1297-1308 (2011).

27. Imaizumi F, Asahina I, Moriyama T, Ishii M, Omura K. Cultured mucosal cell sheet with a double layer of keratinocytes and fibroblasts on a collagen membrane. Tissue Eng. 10(5-6), 657-664 (2004).

28. Mimura T, Yamagami S, Yokoo S et al. Cultured human corneal endothelial cell transplantation with a collagen sheet in a rabbit model. Invest. Ophthalmol. Vis. Sci. 45(9), 2992-2997 (2004).

29. Dekaris I, Gabric N, Mravicic I et al. Multilayer versus monolayer amniotic membrane transplantation for deep corneal ulcer treatment. Coll. Antropol. 25(Suppl.), 23-28 (2001).

30. Owaki T, Shimizu T, Yamato M, Okano T. Cell sheet engineering for regenerative medicine: current challenges and strategies. Biotechnol. J. 9(7), 904-914 (2014).

31. Eyckmans J, Lin GL, Chen CS. Adhesive and mechanical regulation of mesenchymal stem cell differentiation in human bone marrow and periosteum-derived progenitor cells. Biol. Open 1(11), 1058-1068 (2012).

32. Najafabadi MM, Bayati V, Orazizadeh M, Hashemitabar M, Absalan F. Impact of cell density on differentiation efficiency of rat adipose-derived stem cells into schwann-like cells. Int. J. Stem Cells 9(2), 213-220 (2016).

33. Jeon Y, Lee MS, Cheon YP. Decreased contact inhibition in mouse adipose mesenchymal stem cells. Dev. Reprod. 16(4), 329-338 (2012).

34. Kuppers M, Ittrich C, Faust D, Dietrich C. The transcriptional programme of contact-inhibition. J. Cell. Biochem. 110(5), 1234-1243 (2010).

35. Lin CS, Lin G, Lue TF. Allogeneic and xenogeneic transplantation of adipose-derived stem cells in immunocompetent recipients without immunosuppressants. Stem Cells Dev. 21(15), 2770-2778 (2012).

36. Jossen V, Schirmer C, Mostafa Sindi D et al. Theoretical and practical issues that are relevant when scaling up hmsc microcarrier production processes. Stem Cells Int. 2016, 4760414 (2016).

37. Lee KS, Kang HW, Lee HT et al. Sequential sub-passage decreases the differentiation potential of canine adipose-derived mesenchymal stem cells. Res. Vet. Sci. 96(2), 267-275 (2014). 
38. Harris DT. Banking of adipose- and cord tissue-derived stem cells: technical and regulatory issues. Adv. Exp. Med. Biol. 951, 147-154 (2016).

39. Wei S, Wang T, Yonglun L, Guangqian Z. Not all stem cells are equally great: donor single nucleotide polymorphisms (SNP) may matter more than previously thought. Adv. Tissue Eng. Regen. Med. 2(5), 227 (2017). 
